\title{
Oxidative Stress Induced by Epileptic Seizure and Its Attenuation by Melatonin
}

\author{
J. MARE $\check{S}^{1}$, P. STOPKA ${ }^{2}$, K. NOHEJLOVÁ ${ }^{1}$, R. ROKYTA ${ }^{1}$ \\ ${ }^{1}$ Department of Normal Pathological and Clinical Physiology, Third Faculty of Medicine, Charles \\ University in Prague, Czech Republic, ${ }^{2}$ Institute of Inorganic Chemistry, Academy of Sciences \\ of the Czech Republic, Czech Republic
}

Received March 15, 2013

Accepted May 24, 2013

\begin{abstract}
Summary
An epileptic seizure and postictal period in addition to well-known features are also characterized by massive consumption of energy. This is thought to lead to oxidative stress and increased generation of free radicals, which is reflected by increased levels of oxidative products. Our previous work described the neuroprotective effects of melatonin in preventing cognitive worsening after a single epileptic seizure. This work was aimed on direct measurement of free radicals in brain tissue using the EPR method 1, 15 and 60 minutes after seizure. The measurement was performed in adult male Wistar rats at the mentioned intervals after a single tonic-clonic seizure induced by flurothyl. In comparison to control animals there was a significant increase in hydroxyl and nitroxyl radicals 60 minutes after the seizure. The levels of hydroxyl radicals were significantly lower in animals that received melatonin 60 minutes before seizure induction compared to animals without preventive treatment. Therefore, melatonin affected the generation of the measured free radicals differently. An important finding was the delayed increase in free radicals after a single seizure in the later phases of recovery.
\end{abstract}

\section{Key words}

Free radicals $\bullet$ Seizure $\bullet$ Melatonin $\bullet$ EPR

\section{Corresponding author}

Jan Mareš, Department of Normal Pathological and Clinical Physiology, Ke Karlovu 4, 12000 Prague, Czech Republic. Fax: +420 224923 827. E-mail: jan.mares@lf3.cuni.cz

\section{Introduction}

Epileptic seizures markedly increase energy turnover in the central nervous system. Short and long lasting consequences of seizures have been repeatedly described, e.g. post-seizure inhibition (Stripling and Russell 1989), or kindling (Goddard and Douglas 1975). In this relation both production and consumption of energy are increased, especially on the synaptic level of CNS. These processes are associated with development of oxidative stress and generation of large amounts of free radicals (FR). The main sources of FR are mitochondria. Mitochondria are both generators and targets of FR (Folbergrová and Kunz 2012). Changes in morphology of mitochondria were observed immediately after the end of seizure (Langmeier and Mareš 1984). Within 10 minutes mitochondria already returned to normal shape. Mitochondrial damage is also connected to some genetically linked forms of epilepsy (Kunz et al. 2004).

Increase of FR related to seizures is beyond reasonable doubt. Direct influence of FR on long-term potentiation was already described (Jacoby et al. 2001, Lee et al. 2010). Nevertheless, there are almost no studies focusing on the description of changes in FR levels immediately after an acute single seizure.

In experimental epileptology various chemical compounds are used to evoke seizures. It has to be taken in account that convulsiva and there metabolites may influence production of FR. Therefore, use of a volatile convulsant (flurothyl) is advantageous. All seizures elicited by flurothyl mimic naturally occurring ones, acting within tens of seconds of being exhaled without causing morphological injury (Ni et al. 2005). Flurothyl induces seizures avoiding anesthesia and surgery. The mechanism of flurothyl convulsant activity involves 
antagonism of $\mathrm{GABA}_{\mathrm{A}}$ receptor (Krasowski 2000), although, the exact mechanism of its action is not well understood. There is relationship between severe epileptic seizures and increased generation of FR production (de Freitas et al. 2010). Authors suggest that FR plays also important role in the propagation and/or maintenance of convulsive activity. An increase in antioxidant enzymes activities was also mentioned.

Direct exposure to FRs can be reduced by antioxidant application. There is an attention-grabbing beneficial role of the pineal hormone, melatonin, during preventive application. Protective effect of melatonin has been shown in models of cortical ischemic lesions (Deykun et al. 2011) and in preservation of cognitive functions after a single epileptic seizure (Mareš et al. 2012). The strong protective effect of melatonin is thought to be due to its potent antioxidant and FR scavenger properties, both intracellular and extracellular (Reiter 1998).

The aim of the present work was to verify the following hypotheses: 1 . Seizures induced by flurothyl give rise of FRs that could be measured directly using electron paramagnetic resonance spectroscopy (EPR/EPS); 2. Preventive administration of melatonin reduces the amount of free radical levels immediately after the seizure.

The results of the study should also describe the dynamics of changes in FR levels after epileptic seizure and verify the potential for influencing FR levels using the antioxidant and scavenger melatonin.

\section{Methods}

The study was performed on freely moving animals housed under standard conditions.

All procedures were performed in accordance with the Ethical Guidelines of the Third Faculty of Medicine, Charles University, Prague, Czech Republic and in accordance with the Guidelines of the Animal Protection Law of the Czech Republic, which correspond with respective EU regulations. The experimental protocol was approved by the Ethics Committee of the Third Faculty of Medicine, Charles University, Prague, Czech Republic and special care was taken to minimize animal suffering.

\section{Animals and reagents}

Adult, male Wistar rats (ANLAB, Czech Republic), 210-240 g, were used in the experiments. The rats were randomly divided into experimental and control animals. Experimental groups: (1) flurothyl only - F $(n=32)$ was exposed to increasing concentrations of flurothyl vapors until a tonic-clonic seizure was elicited; (2) melatonin + flurothyl - MF $(n=10)$ was pre-treated with melatonin $1 \mathrm{~h}$ before flurothyl seizure induction; (3) tween $80+$ flurothyl - TF $(n=8)$ was pre-treated with tween 80 - melatonin vehicle, $1 \mathrm{~h}$ before flurothyl seizure induction.

Melatonin (Sigma - Aldrich $^{\circledR}$ Inc., Czech Republic) - N-Acetyl-5-methoxytryptamine, was applied i.p. at a dose of $100 \mathrm{mg} / \mathrm{kg}$ in $2 \mathrm{ml}$ of $2 \%$ Tween 80 (polyoxyethylenesorbitan monooleate) solution. A single tonic-clonic seizure was induced by flurothyl (SynQuest Laboratories Inc., USA) - Bis(2,2,2-trifluoroethyl) ether, that was infused using an infusion pump at a of rate $30 \mu \mathrm{l} / \mathrm{min}$. After seizure and prior to decapitation animals were anesthetized with ketamine $100 \mathrm{mg} / \mathrm{kg}$ i.p. and xylazine $16 \mathrm{mg} / \mathrm{kg}$ i.m. (Sigma - Aldrich $^{\circledR}$ Inc., Czech Republic). Extracted brains were homogenized with DMPO (Sigma - Aldrich ${ }^{\circledR}$ Inc., Czech Republic) - 5, 5dimethyl-1-pyroline n-oxide, $100 \mu \mathrm{g}$ per brain - spin trap.

\section{Induction of flurothyl seizures}

The animals from the experimental group $\mathrm{F}$ were exposed to flurothyl, administered via infusion pump, at a constant rate $(30 \mu \mathrm{l} / \mathrm{min})$ while in an air-tight chamber (volume $=14$ liters). The infusion continued onto a filter pad suspended at the top of the chamber, until a tonicclonic seizure was observed. Immediately after the seizure, the flurothyl-air mixture inside the chamber was evacuated and exchanged with fresh air. The animals were immediately removed from the chamber. After recovery from the seizure, the animals were divided into three additional groups to be anesthetized and decapitated at three time intervals - 1 minute (group $1 \mathrm{minF}$ ), 15 minutes (group 15minF) and 60 minutes (group $60 \mathrm{minF}$ ) after the seizure. Brains were removed, homogenized with DMPO and briefly stored in liquid nitrogen until FR measurement. Animals from MF group received melatonin $1 \mathrm{~h}$ prior to induction of seizure. Otherwise group followed the same protocol as the $\mathrm{F}$ group and brains were extracted 60 minutes after the seizure (60minMF).

To ensure that melatonin vehicle Tween 80 has no effect on the levels of free radicals after the seizure group TF was added. Animals from this group receiver Tween $801 \mathrm{~h}$ prior to induction of seizures and were decapitated 60 minutes after the seizure $(60 \mathrm{minTF})$. The 
results were compared with the result of the animals that had induced seizure without any pre-treatment (60minF).

Animals in the control group $(n=20)$ did not undergo any experimental manipulation, were anesthetized, decapitated and underwent FR measurement.

To evaluate behavior during induction of seizures, the following parameters were used: the latencies to the first and total number of oro-facial automatisms (chewing) and myoclonic jerks (i.e. twitches in "Models of Chemically-Induced Acute Seizures" (Velišek 2005)) and latencies of minor (tonic) seizures and the tonic-clonic seizure.

Due to technical and time limitations, experiment was conducted in 4 inconsequent days. The time-line of experiment is presented in schema (Fig. 1). The data from individual days were evaluated separately.

\section{Wistar male rats $(210-240 \mathrm{~g})$}
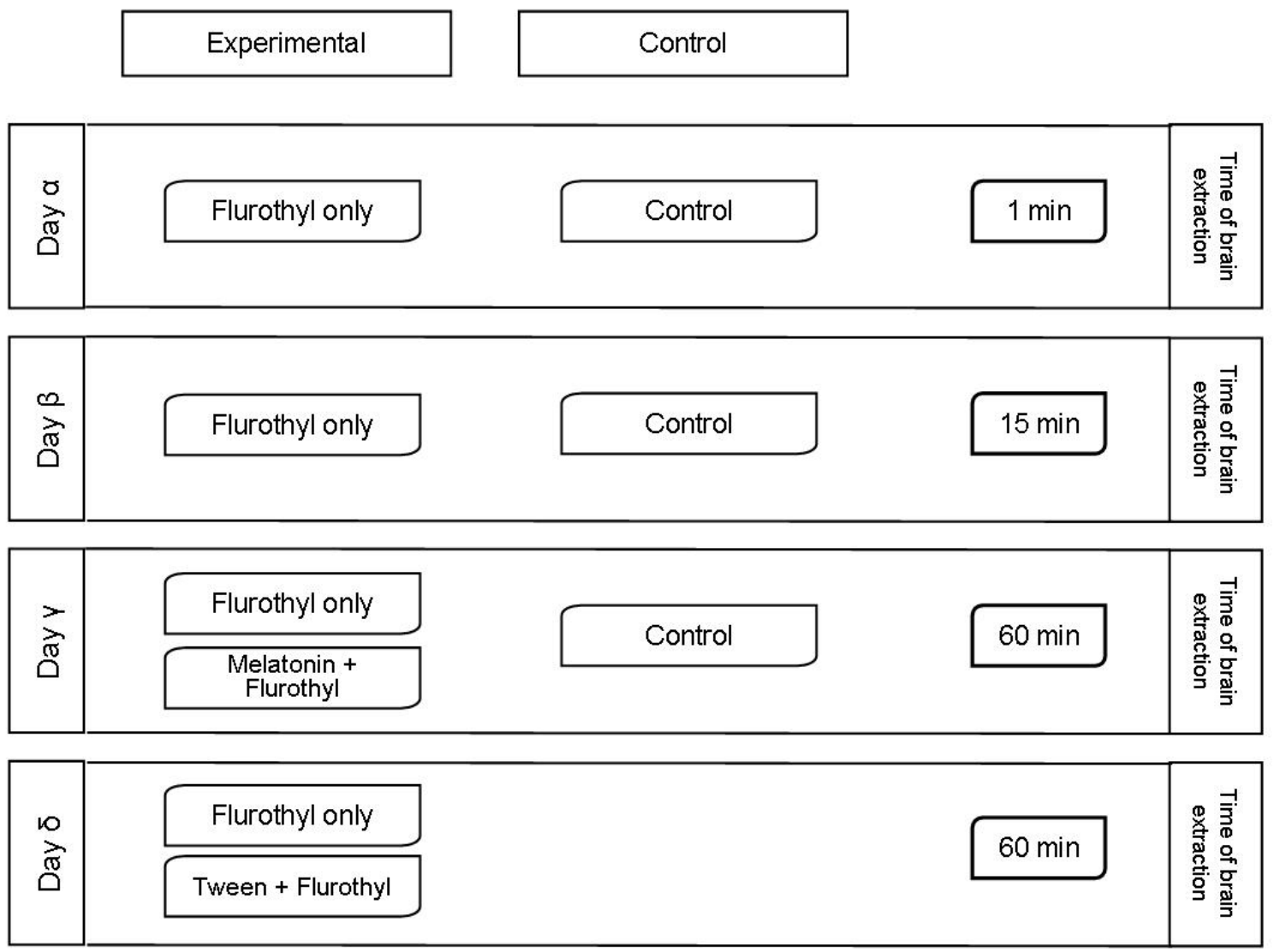

Fig. 1. The schematic time line of experiment. Experiment was conducted in 4 inconsequent days $(a, \beta, y, \delta)$ due to large number of animals and time and technical limitation. Animals underwent assigned manipulation and brains were extracted at certain interval. Measurement of free radicals by EPR followed and each day was evaluated separately. Findings from individual days are incomparable, due to limitations of measurement.

Direct measurement of FR by Electron Paramagnetic (Spin) Resonance Spectroscopy

Measurement of FR (hydroxyl and nitroxyl) levels in brain tissue using electron paramagnetic resonance (EPR) is based on the ability of FRs to absorb microwave energy in strong magnetic fields. Spectra were recorded using an Elexsys E-540 Bruker-Biospin (Rheinstetten, Germany) EPR spectrometer, magnetic fields were evaluated using a 1H-NMR magnetometer and wave frequencies were evaluated with a frequency counter. The EPR spectra were recorded as the first derivation and the main parameters, such as g-factor values, hyperfine coupling constant $\mathrm{A}$, line width $\Delta \mathrm{Hpp}$ (peak-to-peak distance), and $\Delta$ App (peak-to-peak amplitude), were calculated according to Weil and Bolton (1994). The recorded signals were controlled based on the 
spectra of standards $\left(\mathrm{Mn}^{2+} / \mathrm{ZnS}, \mathrm{Cr}^{3+} / \mathrm{MgO}\right.$, Magnettech, Germany). The WINEPR (Bruker, Rheistetten, Germany) software interface was used for spectra recording, handling, and evaluation (Rokyta et al. 2008).

The quantitative data were calculated using double integration or by spectral parameters (Zeman et al. 2009).

\section{Statistical evaluation}

GraphPad Prism 5 (GraphPad Software, Inc., USA) was used for statistical evaluation. All results were tested for normality of distribution using the Kolmogorov-Smirnov test and the $\mathrm{F}$ test was used to

A
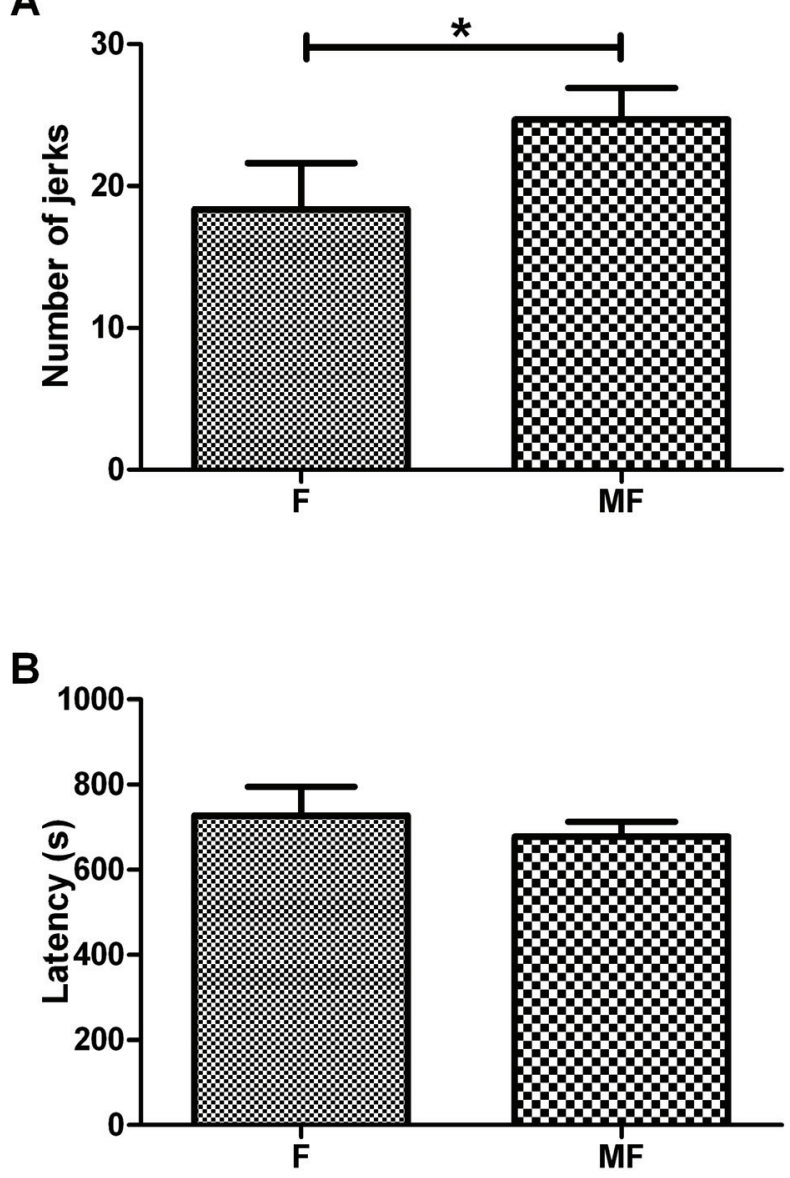

Fig. 2. A. Number of jerks during seizure induction by flurothyl. Data compare $(M \pm S E M)$ number of jerks before occurrence of the tonic-clonic seizure during seizure induction with flurothyl in group F (no treatment) and group MF (melatonin treatment) 60 minutes prior to seizure induction. Asterisk $(*)$ presents statistical analysis: Mann Whitney test, $\mathrm{p}<0.05$. B. Latency of development of tonic-clonic seizures. Data compare $(M \pm S E M)$ latencies of onset of tonic-clonic seizures induced by flurothyl in group $\mathrm{F}$ (no treatment) and group MF (melatonin treatment) 60 minutes prior to seizure induction. There is no significant difference between groups. compare variances.

Results of evaluation of behavior during induction of seizures were analyzed using the Mann Whitney test.

EPR findings of experimental groups $1 \mathrm{minF}$ and $15 \mathrm{minF}$ were compared with the appropriate control group and evaluated using the t-test. Groups $60 \mathrm{minF}$, $60 \mathrm{minMF}$ and $60 \mathrm{minTF}$ were compared with the appropriate control group and analyzed using the oneway ANOVA and Tukey's multiple comparison tests.

Results were accepted as significantly different when $\mathrm{p}<0.05$.

\section{A - ratio of experimental to control values of hydroxyl radicals}

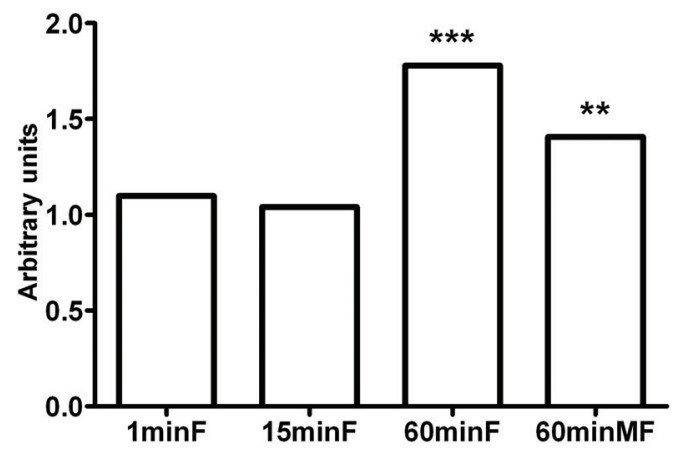

B - ratio of experimental to control values of nitroxyl radicals

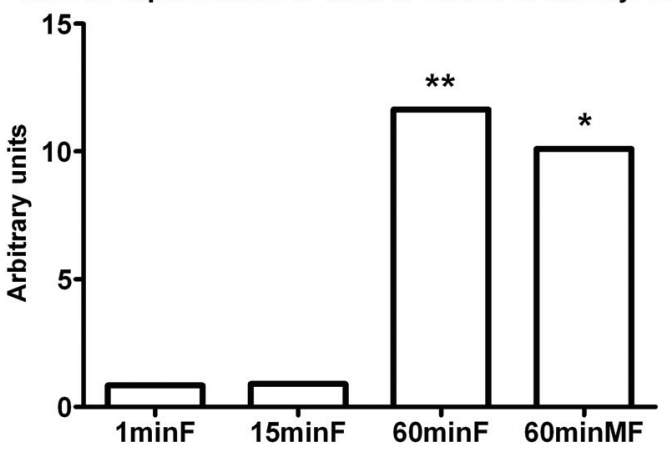

Fig. 3. The dynamic of changes in the levels of free radicals following tonic-clonic seizure. Data present ratio of mean amount of free radicals (hydroxyl radicals - A and nitroxyl radicals - B) in experimental groups and appropriate control groups from the same day of experiment at the certain intervals after seizure development: 1 minute - 1minF, 15 minutes - 15minF, 60 minutes $-60 \mathrm{minF}$ and $60 \mathrm{minMF}$ after the tonic-clonic seizure. Asterisks $(*)$ present statistically significant difference between experimental and appropriate control animals. 


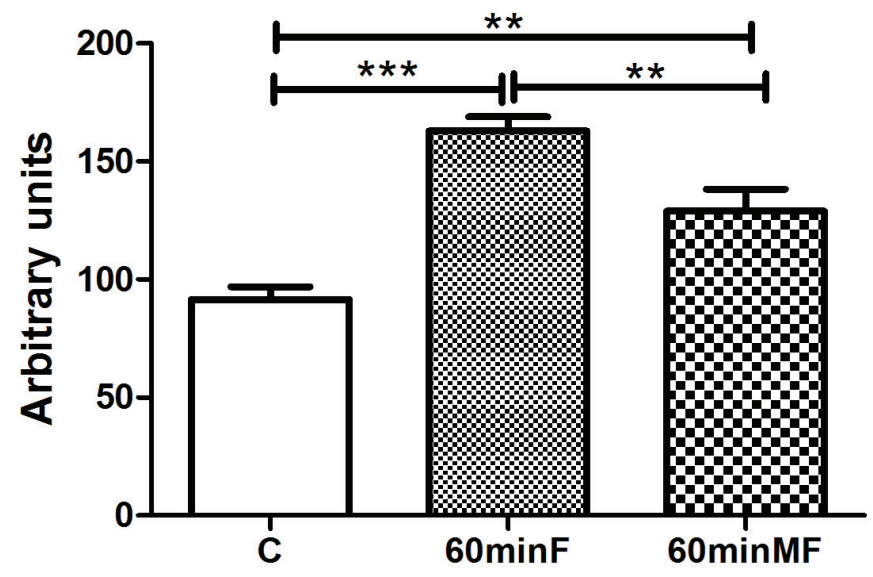

\begin{tabular}{|l|c|c|c|c|c|}
\hline $\begin{array}{l}\text { Tukey's Multiple } \\
\text { Comparison Test }\end{array}$ & Mean Diff. & $\mathrm{q}$ & $\begin{array}{c}\text { Significant? } \\
\mathrm{p}<0.05 ?\end{array}$ & Summary & $95 \%$ Cl of diff \\
\hline C vs 60minF & -71.31 & 10.13 & Yes & $* * *$ & -95.96 to -46.66 \\
\hline C vs 60minMF & -37.30 & 5.177 & Yes & $* *$ & -62.53 to -12.07 \\
\hline 60minF vs 60minMF & 34.01 & 4.831 & Yes & $* *$ & 9.361 to 58.66 \\
\hline
\end{tabular}

Fig. 4. EPR/EPS measurement of hydroxyl radicals $(\bullet \mathrm{OH})$ in brain tissue 60 minutes after seizure induction. Data compares $(M \pm S E M)$ amount of $\bullet \mathrm{OH}$ in arbitrary units. Brains were extracted and stabilized with spin trap DMPO 60 minutes after tonic-clonic seizures. Experimental groups without treatment $(60 \mathrm{minF})$ and with melatonin treatment (60minMF) prior to seizure induction compared with the control group (C). Levels of $\bullet \mathrm{OH}$ in control animals were lower than in both experimental groups. Group with melatonin treatment also had significantly lower amounts of $\bullet \mathrm{OH}$ than the group without treatment. Asterisks present statistical analysis: one way ANOVA with Tukey's post test (see table), $* * \mathrm{p}<0.01, * * * \mathrm{p}<0.001$.

\section{Results}

\section{Evaluation of behavior during seizure induction}

Oro-facial automatism during exposure to flurothyl vapors was only present in $8 \%$ of animals. Its latency of occurrence and number of episodes did not differ between groups. The total number of myoclonic jerks was higher in the MF group (Fig. 2A, $\mathrm{p}<0.05$ ). Latencies of development of minor and tonic-clonic seizures did not significantly differ between groups (Fig. 2B).

\section{Direct measurement of FR by EPR}

There were no significant differences in changes of hydroxyl and nitroxyl radicals levels in brain tissue between experimental and control groups at either 1 minute $(\cdot \mathrm{OH} \mathrm{p}=0.56$; $\mathrm{NO} \bullet \mathrm{p}=0.55)$ or 15 minutes $(\cdot \mathrm{OH}$ $\mathrm{p}=0.88 ; \mathrm{NO} \cdot \mathrm{p}=0.83$ ) after induction.

Elevation of EPR indicated increases in both radicals 60 minutes after seizure induction (Fig. 3).

Seizures induced by flurothyl led to an elevation of hydroxyl radicals (Fig. 4) comparing to the control group (C vs. 60minF; $\mathrm{p}<0.001$ ). Although, application of melatonin prior to seizure induction prevented elevation of hydroxyl radicals (60minF vs. 60minMF; $\mathrm{p}<0.01$ ), there was also significant increase in $\bullet \mathrm{OH}$ radicals in the melatonin treatment group with respect to the control group ( $\mathrm{C}$ vs. $60 \mathrm{minMF}$; $\mathrm{p}<0.01$ ). Levels of $\bullet \mathrm{OH}$ radicals were not affected by the application of Tween 80 (60minF vs. $60 \mathrm{minTF} \mathrm{p}=0.90)$.

Nitroxyl radicals were also elevated by seizures (Fig. 5; C vs. 60minF; $<<0.01$ ), and melatonin treatment did not lower their levels significantly with respect to the control group ( $\mathrm{C}$ vs. 60minMF; $\mathrm{p}<0.05$ ). There was no significant difference between groups with and without melatonin treatment. Levels of NO radicals were not affected by the application of Tween 80 (60minF vs. $60 \operatorname{minTF} \mathrm{p}=0.097$ ).

\section{Discussion}

The results from animals' behavior evaluation during induction of an epileptic seizure using flurothyl were in agreement with our previous findings (Mareš et al. 2012). The group that was pretreated with melatonin had an increased number of jerks when compared to the group without pre-treatment. In contrast to our previous work (Mareš et al. 2012) the latencies to the onset of tonic-clonic seizure in the MF and F groups did not differ significantly. The latencies to the occurrence of the first jerk and time between the first jerk and the start of tonicclonic seizures did not differ either. The latencies to the 
first jerk occurrence also did not differ. The increase in number of jerks in animals treated with melatonin can be explained by an imbalance in the anti- (Sanchez-Forte et al. 1997) and pro-convulsant (Sandyk et al. 1992) mechanisms of activation. The origin/nature of jerks is generally accepted as a manifestation of the activation of neuronal pro-convulsant mechanisms. Whether jerks are an expression of the interplay of above mentioned imbalance of pro-convulsant activity linked to flurothyl and support of anticonvulsant activity by melatonin, remains a question. If this is the case then melatonin increases the number of jerks by affecting activation of one of the system and increasing the imbalance.

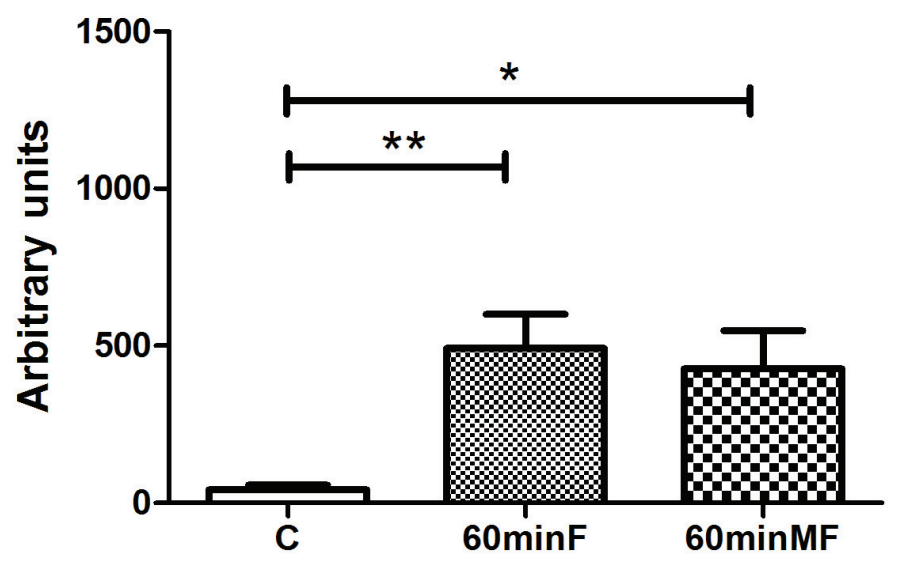

\begin{tabular}{|l|c|c|c|c|c|}
\hline $\begin{array}{l}\text { Tukey's Multiple } \\
\text { Comparison Test }\end{array}$ & Mean Diff. & $\mathrm{q}$ & $\begin{array}{c}\text { Significant? } \\
\mathrm{p}<0.05 ?\end{array}$ & Summary & $95 \% \mathrm{Cl}$ of diff \\
\hline C vs 60minF & -450.0 & 4.748 & Yes & $\star *$ & -781.8 to -118.1 \\
\hline C vs 60min MF & -384.9 & 3.968 & Yes & $*$ & -724.5 to -45.26 \\
\hline $60 \mathrm{minF}$ vs 60minMF & 65.07 & 0.6866 & No & ns & -266.8 to 396.9 \\
\hline
\end{tabular}

Fig. 5. EPR/EPS measurement of nitroxyl radicals (NO•) in the brain tissue 60 minutes after seizure induction. Data compares (Mean \pm SEM) amount of NO• in arbitrary units. Brains were extracted and stabilized with spin trap DMPO 60 minutes after tonic-clonic seizures. Experimental groups without treatment (60minF) and with melatonin treatment (60minMF) prior to seizure induction compared with the control group (C). Levels of NO• in control animals were lower than in both experimental groups. Asterisks present statistical analysis: one way ANOVA with Tukey's post test (see table), $* p<0.05, * * p<0.01$.

The observation that all of the animals from the MF group developed a clonic seizure prior to the tonicclonic seizure, while in group $\mathrm{F}$ approximately one third of the animals progressed to the tonic-clonic seizure without a clonic seizure was accidental. This finding led to further evaluations. First, the difference with in group F relative to the number of animals that had a clonic seizure compared to those that did not, was significant. Second, latencies of clonic seizure development were the same between the F groups and MF groups. Furthermore, differences in latencies of occurrence of clonic seizures did not differ from latencies of direct tonic-clonic seizures in the F group. At the present time we have neither a hypothesis for nor an explanation for these findings. The only conclusion that can be made is that the seizure threshold is the same regardless of severity and it is not affected by melatonin.

FR generation during seizures is already well described. One of the mechanisms relates to mitochondrial injury (Folbergrová and Kunz 2012, Langmeier and Mareš 1984). Another mechanism of FR generation is mediated by activation of glutamate and N-methyl-D-aspartic acid (NMDA) receptors during epileptogenesis (Willmore and Ueda 2009). Non-enzymatic initiation and propagation of oxidative stress eventually leads to lipid peroxidation. It leads to damage to cellular and subcellular membranes and disruption of DNA and amino acids. It is also known that FR increase production of neurotoxic guanidinocompounds, which are endogenous convulsants (Mori et al. 1996). Therefore, application of a scavenger and antioxidant, like melatonin, can impact the pro-convulsant system (Sandyk et al. 1992). Mechanisms of the impact may include three possible explanations. The first and the most prominent mechanism is reinforcement of endogenous antioxidant and scavengers systems. Second involves the dynamics of FR generation. The results of this study demonstrate a significant increase in FR levels one 
hour following the seizure. Earlier measurements, at 1 minute and 15 minutes post-seizure, do not show such an increase. This could be caused by post-ictal mitochondrial "stunning" (i.e. short-lasting, reversible dysfunction), which decreased their capacity for FR generation. In addition, tissue hypoxia that develops during seizure is followed by reoxygenation in the convalescence period. This situation is no doubt similar to ischemia/reperfusion and oxidative stress; and the third mechanism of delayed melatonin effect could be also related to relative hypoxia during the post-ictal period, which could be caused by changes in brain microcirculation, which accompanies post-ictal inhibition.

\section{Conclusions}

The results of present study describe dynamical changes in measured levels of FR (using EPR) in brain tissue following a single epileptic seizure. A large increase in the number of FRs was seen 60 minutes after the seizure, but not immediately after the seizure (1 min and $15 \mathrm{~min}$ ) as was expected. The increases in FRs were significantly reduced when animals were treated with melatonin one hour prior to seizure induction.

\section{Conflict of Interest}

There is no conflict of interest.

\section{Acknowledgements}

This study was supported by the Project of Science Fields Development at Charles University \#PRVOUK P34, University research project \#UNCE-204010 and by the Centralized Development Project \#CSM31/CRP/2013 of the Ministry of Education, Youth and Sports of the Czech Republic.

\section{References}

CHANG SJ, YU BC: Mitochondrial matters of the brain: mitochondrial dysfunction and oxidative status in epilepsy. J Bioenerg Biomembr 42: 457-459, 2010.

DE FREITAS RL, SANTOS IM, DE SOUZA GF, TOME ADA R, SALDANHA GB, DE FREITAS RM: Oxidative stress in rat hippocampus caused by pilocarpine-induced seizures is reversed by buspirone. Brain Res Bull 81: 505-509, 2010.

DEYKUN K, POMETLOVÁ M, SCHUTOVÁ B, MAREŠ J: Modulations of behavioral consequences of minor cortical ischemic lesion by application of free radicals scavengers. Gen Physiol Biophys 30: 263-270, 2011.

FOLBERGROVÁ J, KUNZ WS: Mitochondrial dysfunction in epilepsy. Mitochondrion 12: 35-40, 2012.

GODDARD GV, DOUGLAS RM: Does the engram of kindling model the engram of normal long term memory? Can $J$ Neurol Sci 2: 385-394, 1975.

JACOBY S, SIMS RE, HARTELL NA: Nitric oxide is required for the induction and heterosynaptic spread of longterm potentiation in rat cerebellar slices. $J$ Physiol 535: 825-839, 2001.

KRASOWSKI MD: Differential modulatory actions of the volatile convulsant flurothyl and its anesthetic isomer at inhibitory ligand-gated ion channels. Neuropharmacology 39: 1168-1183, 2000.

KUNZ WS, BIMPONG-BUTA NY, KUDIN AP, ELGER CE: The role of mitochondria in epilepsy: implications for neurodegenerative diseases. Toxicol Mech Methods 14: 19-23, 2004.

LANGMEIER M, MAREŠ J: Changes in some ultrastructural parameters of cortical synapses in the initial phases of kindling. Physiol Bohemoslov 33: 367-375, 1984.

LEE KY, CHUNG K, CHUNG JM: Involvement of reactive oxygen species in long-term potentiation in the spinal cord dorsal horn. J Neurophysiol 103: 382-391, 2010.

MAREŠ J, MAREŠ P, TROJAN S: The ontogenesis of cortical self-sustained after-discharges in rats. Epilepsia 21: 111-121, 1980.

MAREŠ J, POMETLOVÁ M, DEYKUN K, KRÝSL D, ROKYTA R: An isolated epileptic seizure elicits learning impairment which could be prevented by melatonin. Epilepsy Behav 23: 199-204, 2012.

MORI A, KOHNO M, MASUMIZU T, NODA Y, PACKER L: Guanidino compounds generate reactive oxygen species. Biochem Mol Biol Int 40: 135-143, 1996. 
NI H, JIANG YW, BO T, WANG JM, WU XR: c-Fos, N-methyl-d-aspartate receptor 2C, GABA-A-alpha1 immonoreactivity, seizure latency and neuronal injury following single or recurrent neonatal seizures in hippocampus of Wistar rat. Neurosci Lett 380: 149-154, 2005.

REITER RJ: Oxidative damage in the central nervous system: protection by melatonin. Prog Neurobiol 56: 359-384, 1998.

ROKYTA R, STOPKA P, KÁFUŇKOVÁ E, KŘÍŽOVÁ J, FRICOVÁ J, HOLEČEK V: The evaluation of nociceptive intensity by using free radicals direct measurement by EPR method in the tail of anaesthetized rats. Neuro Endocrinol Lett 29: 1007-1014, 2008.

SANCHEZ-FORTE M, MORENO-MADRID F, MUNOZ-HOYOS A, MOLINA-CARBALLO A, ACUNACASTROVIEJO D, MOLINA-FONT JA: The effect of melatonin as anti-convulsant and neuron protector. (in Spanish) Rev Neurol 25: 1229-1234, 1997.

SANDYK R, TSAGAS N, ANNINOS PA: Melatonin as a proconvulsive hormone in humans. Int J Neurosci 63: 125 $135,1992$.

STRIPLING JS, RUSSELL RD: Twenty-four-hour post-seizure inhibition during limbic kindling requires seizure generalization. Neurosci Lett 99: 208-213, 1989.

VELIŠEK L: Models of chemically-induced acute seizures. In: Models of Seizures and Epilepsy. A PITKANEN, PA SCHWARTZKRONIN, SL MOSHE (eds), Academic Press, Waltham, 2005, pp 127-152.

WEIL JA, BOLTON JR: Electron Paramagnetic Resonance. Elementary Theory and Practical Application. J. Wiley and Sons, New York, 1994.

WILLMORE LJ, UEDA Y: Posttraumatic epilepsy: hemorrhage, free radicals and the molecular regulation of glutamate. Neurochem Res 34: 688-697, 2009.

ZEMAN M, STOPKA P, VECKA M, ŽÁK A, PÍSǍ̌íKOVÁ A, JIRÁK R, STAŇKOVÁ B, VÁVROVÁ L, KODYDKOVÁ J, KŘÍŽOVÁ J, MACÁŠEK J: Electron spin resonance determination of hydroxyl and nitroxide radicals in depressions and hyperlipidemia. (in Czech) Chemicke listy 103: 667-671, 2009. 